\section{Tödliche Anaphylaxien in USA meist iatrogen}

Die Zahl der Anaphylaxie-bedingten Todesfälle ist in den USA relativ stabil. Bei den Ursachen hat es jedoch eine deutliche Verschiebung gegeben. Dies hat kürzlich eine US-amerikanische Untersuchung ergeben.

$\ddot{A}$ rzte des Albert Einstein College of Medicine (Bronx/New York) haben untersucht, wie sich Häufigkeit und Ursachen von tödlichen Anaphylaxien in den USA seit 1999 gewandelt haben. Grundlage ihrer Analyse waren die laut nationalem Sterberegister im Totenschein dokumentierten ICD-10-Diagnosen.

In den Jahren 1999-2010 wurde 2.458mal eine Anaphylaxie als Todesursache festgestellt. Das entspricht einer Prävalenz von 0,69 pro einer Millionen Menschen. Die zugrunde liegende anaphylaktische Reaktion war in 58,8 \% der Fälle durch ein Medikament ausgelöst worden. Auf den Plätzen zwei und drei folgten nicht näher spezifizierte Anaphylaxien mit 19,3\% und Reaktionen auf
Insektengift mit 15,2\%. Nahrungsmittelanaphylaxien hatten mit $6,7 \%$ den geringsten Anteil.

Letale Anaphylaxien durch Medikamente haben sich im Untersuchungszeitraum fast verdoppelt, von 0,27 auf 0,51 pro einer Millionen Menschen. Ältere Menschen waren verstärkt betroffen: Bei den über 80-Jährigen betrug die Quote 1,28 , bei den unter 20 -Jährigen nur 0,05 , wiederum jeweils pro einer Millionen Menschen. Allerdings war nur in jedem vierten Fall der auslösende Wirkstoff dokumentiert. Hier lagen Antibiotika mit $40,5 \%$ vorne, gefolgt von Röntgenkontrastmitteln plus anderen Diagnostika $(30,4 \%)$ und Krebsmedikamenten $(12,5 \%)$. Sonstige Auslöser waren Blut- drucksenker, Opioide, nicht steroidale Antirheumatika, Seren und Anästhetika.

Tödliche Anaphylaxien durch Insektenstiche und Lebensmittel blieben auf einem konstanten Niveau. Sie ereigneten sich ebenfalls öfter in höherem Alter und waren zudem bei Männern häufiger. Die Zahl der Anaphylaxie-bedingten Todesfälle mit nicht identifiziertem Auslöser ging dagegen zurück.

Fazit: Laut den Ergebnissen dieser Studie verursachen in den USA Medikamente die meisten anaphylaktischen Reaktionen mit tödlichem Ausgang. Die Studienautoren um Elina Jerschow machen dafür neben Veränderungen in der Diagnosekodierung auch den steigenden Einsatz von Medikamenten sowie Röntgenkontrastmitteln verantwortlich.

Dr. Beate Schumacher

Jerschow E et al. Fatal anaphylaxis in the United States, 1999-2010: Temporal patterns and demographic associations. J Allergy Clin Immunol 2014; pii: S0091-6749(14)01190-7 